\title{
Extension of lacunary statistical convergence on vector valued double difference sequence space
}

\author{
Anindita Basu \\ Department of Mathematics, Dr. B. N. Dutta Smriti Mahavidyalaya, Burdwan-713407, West Bengal, India \\ E-mail: anindita.basu10@gmail.com
}

\begin{abstract}
In this paper the concepts of lacunary vector valued double sequences and $\Delta_{11}$-lacunary statistical convergent vector valued double difference sequences have been introduced. Further, the purpose of this work is to extend the known sequence space in the literature for ordinary single sequences to the double sequence space $\Delta_{11} N_{\theta_{r, s}}(E)$ of lacunary strongly convergent vector valued double sequences. Some inclusion relations among them are also established. Lastly, this paper deals with some results which establish the relationship between various lacunary methods.
\end{abstract}

2000 Mathematics Subject Classification. 46A45. 46B45.

Keywords. Lacunary double sequences, statistical convergence, lacunary refinement.

\section{Introduction \& Motivation}

The idea of statistical convergence was given by Zygmund [5] in the fitst edition of his monograph in 1935. The concept of statistical convergence for single sequence of real or complex numbers has been first introduced by H. Fast [14], Buck [22] and Schoenberg [15] independently. From the point of view of sequence spaces, this concept has been generalized \& developed by Fridy [19], Maddox [17], Salat [23], Connor [18], Rath \& Tripathy [6] and many others.

The basic concept of statistical convergence is based on the notion of natural density or asymptotic density [16] of a set.

The scalar sequence $\mathrm{x}=\left(x_{k}\right)$ is said to be statistically convergent to the number $\ell$, if for each $\varepsilon>0$, the natural density

$$
\delta\left(\left\{k \leq n:\left|x_{k}-\ell\right| \geq \varepsilon\right\}\right)=0
$$

and it is denoted as $x_{k} \longrightarrow \ell\left(s t_{1}\right)$, while Fridy [19] defined a sequence $x=\left(x_{k}\right)$ to be statistically Cauchy, if for every $\varepsilon>0$, there exists a number $\mathrm{N}=N(\varepsilon)$ such that

$$
\delta\left(\left\{k \leq n:\left|x_{k}-x_{N}\right| \geq \varepsilon\right\}\right)=0 .
$$

The space of lacunary strong convergence has been defined as follows:

A sequence of positive integers $\theta=\left(k_{r}\right)$ is called "lacunary" if $k_{0}=0,0<k_{r}<k_{r+1}$ and $h_{r}=k_{r}-k_{r-1} \rightarrow \infty$, as $r \rightarrow \infty$. The intervals determined by $\theta$ are denoted by $I_{r}=\left(k_{r-1, k_{r}}\right]$ and the ratio $\frac{k_{r}}{k_{r-1}}$ will be denoted by $q_{r}$. The space of lacunary strongly convergent sequences $N_{\theta}$ is defined by Freedman et. al. [4] as follows:

$$
N_{\theta}=\left\{x=\left(x_{i}\right): \lim _{r \rightarrow \infty} h_{r}^{-1} \sum_{i \in I_{r}}\left|x_{i}-s\right|=0, \text { for some s }\right\} .
$$


Fridy and Orhan ([10], [11]) combined the concepts of statistical convergence and lacunary convergence and introduced a new convergence method, known as lacunary statistical convergence or $S_{\theta}$-convergence for single scalar sequences as follows:

A sequence $\mathrm{x}=\left(x_{k}\right)$ is said to be $S_{\theta}$-convergent to $\mathrm{L}$ if for each $\varepsilon>0$,

$$
\lim _{r \rightarrow \infty} \frac{1}{h_{r}} C\left(\left\{k \in I_{r}:\left|x_{k}-L\right| \geq \varepsilon\right\}\right)=0
$$

where $\theta=\left(k_{r}\right)$ is a lacunary sequence and $h_{r}=k_{r}-k_{r-1}$ and $C(A)$ denotes the number of elements in the enclosed set $\mathrm{A}$. In this case, we write $x_{k} \longrightarrow \ell\left(S_{\theta}\right)$.

Recently Bilgin [24], Jinlu Li [20], Karakaya et al. [12], Çakalli [8], Basu et al. ([1], [2]), Savas et al. [7] and many others have extended the concept of lacunary statistical convergence and continued this study by introducing new sequence spaces using modulus functions, Orlicz functions, infinite matrices etc.

Mursaleen et al. [13] first introduced and extended the concept of statistical convergence for double sequences of real or complex numbers after defining the analogue concept of natural density for double sequences as follows:

Let $\mathrm{K} \subseteq \mathbb{N} \times \mathbb{N}$ be a two dimensional set of positive integers and let

$$
K(n, m)=C(\{(i, j): i \leq n \text { and } j \leq m\}) .
$$

where $\mathrm{C}(\mathrm{A})$ denotes the cardinality of the set $\mathrm{A}$.

If the sequence $\left(\frac{K(n, m)}{n m}\right)$ has a limit in Pringsheim's sense [3], then we say that $\mathrm{K}$ has double natural density $\delta_{2}(K)$ and is written as

$$
\delta_{2}(K)=P-\lim _{n, m} \frac{K(n, m)}{n m}
$$

Mursaleen et al. [13] defined analogously the statistical convergence and statistical Cauchy convergence for double sequences $\mathrm{x}=\left(x_{n k}\right)$ as follows:

Definition 1.1. A real double sequence $x=\left(x_{i j}\right)$ is said to be statistically convergent to the number $\ell$, if for each $\varepsilon>0$, the set

$$
\left\{(i, j): i \leq n \text { and } \mathrm{j} \leq \mathrm{m}:\left|\mathrm{x}_{\mathrm{ij}}-\ell\right| \geq \varepsilon\right\}
$$

has double natural density zero in the Pringsheim's sense, i.e.,

$$
P-\lim _{m, n} \frac{1}{m n} C\left(\left\{(i, j): i \leq n \& j \leq m,\left|x_{i j}-\ell\right| \geq \varepsilon\right\}\right)=0
$$

and this is denoted as st $\lim _{i, j} x_{i j}=\ell$. We denote the set of all statistically convergent sequences (in Pringsheim's sense) by st . .

Definition 1.2. A real double sequence $x=\left(x_{i j}\right)$ is said to be statistically Cauchy, if for each $\varepsilon>0$ there exist $A=A(\varepsilon)$ and $B=B(\varepsilon)$ such that for all $i, p \geq A, j, q \geq B$, the set

$$
\left\{(i, j): i \leq r \text { and } \mathrm{j} \leq \mathrm{s}:\left|\mathrm{x}_{\mathrm{ij}}-\mathrm{x}_{\mathrm{pq}}\right| \geq \varepsilon\right\}
$$

has double natural density zero in Pringsheim's sense. 


\section{Concepts of $\Delta_{11}$-lacunary statistically convergent \& Cauchy double sequences}

We first introduce the concept of lacunary double sequences as follows:

Definition 2.1. By a lacunary double sequence we mean an increasing sequence of positive integers $\theta_{r, s}=\left(k_{r s}\right)$

$$
\begin{array}{llllllll}
k_{00} & k_{01} & \ldots & \ldots & \ldots & \ldots & \ldots & \ldots \\
k_{10} & k_{11} & k_{12} & \ldots & \ldots & \ldots & \ldots & \ldots \\
k_{20} & k_{21} & k_{22} & k_{23} & \ldots & \ldots & \ldots & \ldots \\
k_{30} & k_{31} & k_{32} & k_{33} & k_{34} & \ldots & \ldots & \ldots \\
k_{40} & k_{41} & k_{42} & k_{43} & k_{44} & k_{45} & \ldots & \ldots \\
k_{50} & k_{51} & k_{52} & k_{53} & k_{54} & k_{55} & k_{56} & \ldots \\
\ldots & \ldots & \ldots & \ldots & \ldots & \ldots & \ldots & \ldots
\end{array}
$$

such that $k_{00}=0, k_{-1} 0=0, k_{0-1}=0, k_{r 0}=0, k_{0 s}=0$ for $r \geq 1, s \geq 1$,

$$
\begin{aligned}
& 0<k_{m j}<k_{n j} \text { if } m<n \\
& 0<k_{i m}<k_{\text {in }} \text { if } m<n, \\
& 0<k_{i j}<k_{i+1} 1, \text { for } i=1,2, \ldots \text { and } \mathrm{j}=1,2, \ldots
\end{aligned}
$$

and $\Delta_{01} h(r, s)=\left(k_{r s}-k_{r s-1}\right) \rightarrow \infty$ as $s \rightarrow \infty, \Delta_{10} h(r, s)=\left(k_{r s}-k_{r-1, s}\right) \rightarrow \infty$ as $r \rightarrow \infty$. The corresponding intervals are denoted by $\Delta_{01} I(r, s)$ and $\Delta_{10} I(r, s)$ where $\Delta_{01} I(r, s)=\left(k_{r} s-1, k_{r s}\right]$ and $\Delta_{10} I(r, s)=\left(k_{r-1, s}, k_{r s}\right]$.

We define

$$
\Delta h_{r s} \equiv \Delta_{10} h(r, s) \times \Delta_{01} h(r, s)=\underbrace{\left(k_{r s}-k_{r-1} s\right)}_{\Delta_{10} h(r, s)} \times \underbrace{\left(k_{r s}-k_{r s-1}\right)}_{\Delta_{01} h(r, s)} .
$$

So $\Delta h_{r s} \rightarrow \infty$ as $r, s \rightarrow \infty$.

The intervals determined by $\theta_{r, s}$ are denoted by $\Delta I_{r s}$ where

$$
\Delta I_{r s} \equiv \Delta_{10} I(r, s) \times \Delta_{01} I(r, s)=\underbrace{\left(\begin{array}{llll}
k_{r-1} s, & k_{r} & s
\end{array}\right]}_{\Delta_{10} I(r, s)} \times \underbrace{\left(\begin{array}{lll}
k_{r} s-1 & , & k_{r} s
\end{array}\right]}_{\Delta_{01} I(r, s)}
$$

and the ratios $\frac{k_{r s}}{k_{r-1, s}}, \frac{k_{r s}}{k_{r s-1}}$ are denoted by $\Delta_{10} q_{r s}$ and $\Delta_{01} q_{r s}$ respectively. We will denote the set of all double lacunary sequences by $N_{\theta_{r, s}}$. follows:

Recently Edely et al. [21] defined the backward differences of the double sequences $\mathrm{x}=\left(x_{n k}\right)$ as

$$
\begin{aligned}
& \Delta_{11} x_{n k}=x_{n k}-x_{n-1 k}-x_{n k-1}+x_{n-1 k-1}, \\
& \Delta_{10} x_{n k}=x_{n k}-x_{n-1 k} \\
& \Delta_{01} x_{n k}=x_{n k}-x_{n k-1} .
\end{aligned}
$$

We now define $\Delta_{11}$-statistical convergence, $\Delta_{11}$-lacunary statistical convergence, $\Delta_{11}$-lacunary statistically Cauchy sequence for double sequences in the following manner: 
Definition 2.2. A double sequence $x=\left(x_{i j}\right)$, where $x_{i j} \in E$, is said to be $\Delta_{11}$-statistical convergent to $\ell \in E$ if, for each $\varepsilon>0$, the set

$$
\left\{(i, j), i \leq n \text { and } j \leq m:\left\|\Delta_{11} x_{i j}-\ell\right\|_{E} \geq \varepsilon\right\}
$$

has double natural density zero, where, the convergence is in Pringsheim's sense. i.e.,

$$
P-\lim _{m, n} \frac{1}{m n} C\left(\left\{(i, j): i \leq n \& j \leq m,\left\|\Delta_{11} x_{i j}-\ell\right\| \geq \varepsilon\right\}\right)=0 .
$$

Definition 2.3. Let $\theta_{r, s}$ be a double lacunary sequence. A double sequence $x=\left(x_{i j}\right)$, where $x_{i j} \in E$, a Banach space, is said to be $\Delta_{11}$-lacunary statistical convergent to $\ell \in E$ or $\Delta_{11}-S_{\theta_{r, s}}$ convergent to $\ell \in E$, if for each $\varepsilon>0$,

$$
P-\lim _{r, s} \frac{1}{\Delta h_{r s}} C\left(\left\{(i, j) \in \Delta I_{r s}:\left\|\Delta_{11} x_{i j}-\ell\right\|_{E} \geq \varepsilon\right\}\right)=0 .
$$

We denote it as $x_{n k} \stackrel{P}{\longrightarrow} \ell\left(\Delta_{11}-S_{\theta_{r, s}}\right)$.

Definition 2.4. Let $\theta_{r, s}$ be a double lacunary sequence. A double sequence $x=\left(x_{i j}\right), x_{i j} \in E$, a Banach space, is said to be $\Delta_{11}$-lacunary statistically Cauchy sequence or $\Delta_{11}-S_{\theta_{r, s}}$ Cauchy if there is a subsequence $\left\{x_{i^{\prime}(r)} j^{\prime}(s)\right\}$ of $x$ such that $\left(i^{\prime}(r), j^{\prime}(s)\right) \in \Delta I_{r s}$ for each $r$ and $s$ and

$$
\lim _{r, s \rightarrow \infty} x_{i^{\prime}(r)} j^{\prime}(s) \stackrel{P}{=} \ell
$$

for some $\ell \in E$ and for every $\varepsilon>0$,

$$
P-\lim _{r, s} \frac{1}{\Delta h_{r s}} C\left(\left\{(n, k) \in \Delta I_{r s}:\left\|\Delta_{11} x_{i j}-\Delta_{11} x_{i^{\prime} r} j^{\prime}(s)\right\|_{E} \geq \varepsilon\right\}\right)=0 .
$$

\section{New sequence space $\Delta_{11} N_{\theta_{r, s}}(E)$}

We define the new class of lacunary strongly vector valued convergent double sequences as follows:

Let $\theta_{r, s}=\left(k_{r s}\right)$ be a lacunary double sequence. Let $\left(\mathrm{E},\|.\|_{E}\right)$ be a Banach space over the field of complex number $\mathbb{C}$. The class $\Delta_{11} N_{\theta_{r, s}}(E)$ of lacunary strongly convergent vector valued double sequences is defined as

$$
\begin{array}{r}
\Delta_{11} N_{\theta_{r, s}}(E)=\left\{x=\left(x_{n k}\right)_{n, k=0}^{\infty}: x_{n k} \in E \text { and there exist } \ell \in E\right. \text { such that } \\
\left.P-\lim _{r, s} \frac{1}{\Delta h_{r s}} \sum_{(n, k) \in \Delta I_{r s}}\left\|\Delta_{11} x_{n k}-\ell\right\|_{E}=0\right\}
\end{array}
$$

where $\Delta_{11} x_{n k}$ is defined in (2.1).

The space of all lacunary convergent null double sequences is denoted by $\Delta_{11} N_{\theta_{r, s}}^{0}(E)$.

Note 3.1. If $x=\left(x_{n k}\right) \in \Delta_{11} N_{\theta_{r, s}}(E)$ we denote this as $x_{n k} \longrightarrow \ell\left(N_{\theta_{r, s}}\right)$.

Now we have 
Theorem 3.1. $\Delta_{11} N_{\theta_{r, s}}^{0}(E)$ is a Banach space with respect to the norm

$$
\begin{aligned}
\left\|x_{n k}\right\|_{E}\left(\theta_{r, s}\right)=\left\|x_{00}\right\|_{E}+\left\|x_{01}\right\|_{E} & +\sup _{r, s} \frac{1}{\Delta h_{r s}}\left[\sum_{(n, k) \in \Delta I_{r s}}\left\|x_{n k}-x_{n-1}\right\|_{E}\right. \\
& \left.+\sum_{(n, k) \in \Delta I_{r s}}\left\|x_{n k-1}-x_{n-1} k-1\right\|_{E}\right]
\end{aligned}
$$

Proof. The proof is easy, so we omit it.

Q.E.D.

\section{Some Results}

Theorem 4.1. The sequence $x=\left(x_{n k}\right), x_{n k} \in E$ is $\Delta_{11}$-lacunary statistically convergent if and only if $x=\left(x_{n k}\right)$ is $\Delta_{11}$-lacunary statistically Cauchy sequence. Equivalently, the sequence $x=\left(x_{n k}\right)$ is $\Delta_{11}-S_{\theta_{r, s}}$-convergent if and only if $x=\left(x_{n k}\right)$ is $\Delta_{11}-S_{\theta_{r, s}}$ Cauchy sequence.

Proof. Let us assume that $\mathrm{x}=\left(x_{n k}\right)$ is $\Delta_{11}$-lacunary statistically Cauchy sequence. Then there is a subsequence $\left\{x_{n^{\prime}(r)} k^{\prime}(s)\right\}$ of $\mathrm{x}$ such that $\left(n^{\prime}(r), k^{\prime}(s)\right) \in \Delta I_{r s}$, for each $\mathrm{r}$ and $\mathrm{s}$, and $P-$ $\lim _{r, s \rightarrow \infty} x_{n^{\prime}(r) k^{\prime}(s)}=\ell$ for some $\ell \in E$ and for every $\varepsilon>0$,

$$
P-\lim _{r, s \rightarrow \infty} \frac{1}{\Delta h_{r s}} C\left(\left\{(n, k) \in \Delta I_{r s}:\left\|\Delta_{11} x_{n k}-\Delta_{11} x_{n^{\prime}(r)} k^{\prime}(s)\right\|_{E} \geq \varepsilon\right\}\right)=0 .
$$

So for every $\varepsilon>0$,

$$
\begin{aligned}
& C\left(\left\{(n, k) \in \Delta I_{r s}:\left\|\Delta_{11} x_{n k}-\ell\right\|_{E} \geq \varepsilon\right\}\right) \\
\leq & C\left(\left\{(n, k) \in \Delta I_{r s}:\left\|\Delta_{11} x_{n k}-\Delta_{11} x_{n^{\prime}(r) k^{\prime}(s)}\right\|_{E} \geq \frac{\varepsilon}{2}\right\}\right) \\
+ & C\left(\left\{(n, k) \in \Delta I_{r s}:\left\|\Delta_{11} x_{n^{\prime}(r) k^{\prime}(s)}-\ell\right\|_{E} \geq \frac{\varepsilon}{2}\right\}\right) .
\end{aligned}
$$

Now dividing both sides by $\Delta h_{r s}$ and taking P-limit we get

$$
P-\lim _{r, s} \frac{1}{\Delta h_{r s}} C\left(\left\{(n, k) \in \Delta I_{r s}:\left\|\Delta_{11} x_{n k}-\ell\right\|_{E} \geq \varepsilon\right\}\right)=0 .
$$

Hence it follows that $\mathrm{x}=\left(x_{n k}\right)$ is $\Delta_{11}$-lacunary statistically convergent to $\ell$.

Conversely, assume that $\mathrm{x}=\left(x_{n k}\right)$ is $\Delta_{11}$-lacunary statistically convergent to $\ell$.

Then by definition, for each natural number $\alpha$,

$$
P-\lim _{r, s \rightarrow \infty} \frac{1}{\Delta h_{r s}} C\left(\left\{(n, k) \in \Delta I_{r s}:\left\|\Delta_{11} x_{n k}-\ell\right\|_{E}<\frac{1}{\alpha}\right\}\right)=1 .
$$

Let us choose $\mathrm{m}(1), \mathrm{p}(1)$ such that for $r \geq m(1)$ and $s \geq p(1)$ imply

$$
\frac{1}{\Delta h_{r s}} C\left(\left\{(n, k) \in \Delta I_{r s}:\left\|\Delta_{11} x_{n k}-\ell\right\|_{E}<1\right\}\right)>0
$$

i. e.,

$$
\left\{(n, k) \in \Delta I_{r s}:\left\|\Delta_{11} x_{n k}-\ell\right\|_{E}<1\right\} \neq \varphi
$$


Next we choose $m(2)>m(1)$ and $p(2)>p(1)$ such that $r \geq m(2)$ and $s \geq p(2)$ imply

$$
\frac{1}{\Delta h_{r s}} C\left(\left\{(n, k) \in \Delta I_{r s}:\left\|\Delta_{11} x_{n k}-\ell\right\|_{E}<\frac{1}{2}\right\}\right)>0 \text {. }
$$

Then for each $\mathrm{r}$ and s satisfying $m(1) \leq r<m(2)$ and $p(1) \leq s<p(2)$ respectively choose $\left(n^{\prime}(r) k^{\prime}(s)\right) \in \Delta I_{r s}$ such that

$$
\left\|\Delta_{11} x_{n^{\prime}(r)} k^{\prime}(s)-\ell\right\|_{E}<1 .
$$

Generalizing this we get, if we choose $m(\nu+1)>m(\nu)$ and $p(\nu+1)>p(\nu)$ such that $r \geq m(\nu+1)$ and $s \geq p(\nu+1)$ imply

$$
\left\{\left(n^{\prime}(r), k^{\prime}(s)\right) \in \Delta I_{r s}:\left\|\Delta_{11} x_{n^{\prime}(r)} k^{\prime}(s)-\ell\right\|_{E}<\frac{1}{\nu+1}\right\} \neq \varphi .
$$

Hence for all $\mathrm{r}$ and s satisfying $m(\nu) \leq r<m(\nu+1)$ and $p(\nu) \leq s<p(\nu+1)$ we choose $\left(n^{\prime}(r), k^{\prime}(s)\right) \in \Delta I_{r s}$ such that

$$
\left\|\Delta_{11} x_{n^{\prime}(r) k^{\prime}(s)}-\ell\right\|_{E}<\frac{1}{\nu} .
$$

Hence we get $\left(n^{\prime}(r), k^{\prime}(s)\right) \in \Delta I_{r s}$ for every $\mathrm{r}$ and $\mathrm{s}$ and (4.1) implies that

$$
P-\lim _{r, s \rightarrow \infty} x_{n^{\prime}(r)} k^{\prime}(s)=\ell \text {. }
$$

Consequently for every $\varepsilon>0$,

$$
\begin{aligned}
& \frac{1}{\Delta h_{r s}} C\left(\left\{(n, k) \in \Delta I_{r s}:\left\|\Delta_{11} x_{n k}-\Delta_{11} x_{n^{\prime}(r) k^{\prime}(s)}\right\|_{E} \geq \varepsilon\right\}\right) \\
\leq & \frac{1}{\Delta h_{r s}} C\left(\left\{(n, k) \in \Delta I_{r s}:\left\|\Delta_{11} x_{n k}-\ell\right\|_{E} \geq \frac{\varepsilon}{2}\right\}\right) \\
+ & \frac{1}{\Delta h_{r s}} C\left(\left\{(n, k) \in \Delta I_{r s}:\left\|\Delta_{11} x_{n^{\prime}(r) k^{\prime}(s)}-\ell\right\|_{E} \geq \frac{\varepsilon}{2}\right\}\right) .
\end{aligned}
$$

Since $\mathrm{x}=\left(x_{n k}\right)$ is $\Delta_{11}$-lacunary statistically convergent to $\ell$ and $\lim _{r, s \rightarrow \infty} x_{n^{\prime}(r) k^{\prime}(s)}=\ell$, it follows that $\mathrm{x}=\left(x_{n k}\right)$ is $\Delta_{11}$-lacunary statistically Cauchy sequence.

Q.E.D.

Theorem 4.2. Let $\theta=\left\{k_{r s}\right\}$ be a lacunary sequence, then

(a) $x_{n k} \stackrel{P}{\longrightarrow} \ell\left(\Delta_{11}-N_{\theta_{r, s}}\right)$ implies $\left.x_{n k} \stackrel{P}{\longrightarrow} \ell\left(\Delta_{11}-S_{\theta_{r, s}}\right)\right)$. But the converse is not true.

(b) $x \in \ell_{\infty}\left(\Delta_{11}\right)$ and $x_{n k} \stackrel{P}{\longrightarrow} \ell\left(\Delta_{11}-S_{\theta_{r, s}}\right)$ imply $x_{n k} \stackrel{P}{\longrightarrow}\left(\Delta_{11}-N_{\theta_{r, s}}\right)$, where

$$
\ell_{\infty}\left(\Delta_{11}\right)=\left\{x=\left(x_{n k}\right): x_{n k} \in E, \exists \ell \in E \text { such that }\left\|\Delta_{11} x_{n k}-\ell\right\|_{E} \leq M \text { for all } n, k\right\}
$$

Proof. (a) If $\varepsilon>0$ and $x_{n k} \stackrel{P}{\longrightarrow} \ell\left(\Delta_{11}-N_{\theta_{r, s}}\right)$, we can write

$$
\begin{aligned}
\sum_{(n, k) \in \Delta I_{r s}}\left\|\Delta_{11} x_{n k}-\ell\right\|_{E} \geq & \sum_{\substack{(n, k) \in \Delta I_{r s} \\
\left\|\Delta_{11} x_{n k}-\ell\right\|_{E} \geq \varepsilon}}\left\|\Delta_{11} x_{n k}-\ell\right\|_{E} \\
& \geq \varepsilon C\left(\left\{(n, k) \in \Delta I_{r s}:\left\|\Delta_{11} x_{n k}-\ell\right\|_{E} \geq \varepsilon\right\}\right),
\end{aligned}
$$


Dividing by $\Delta h_{r s}$ and taking the P-limit as $\mathrm{r}, \mathrm{s} \rightarrow \infty$ we get the first part of (a) as

$$
P-\lim _{r, s} \frac{1}{\Delta h_{r s}} \sum_{(n, k) \in \Delta I_{r s}}\left\|\Delta_{11} x_{n k}-\ell\right\|_{E}=0 .
$$

To prove the other part we assume that $\theta_{r, s}=\left(k_{r s}\right)$ is a lacunary sequence and $\bar{\theta}$ is the null element of the Banach space E.

Let us define a double sequence $x=\left(x_{n k}\right)$ such that

\begin{tabular}{|c|c|c|c|c|c|c|}
\hline$\Delta_{11} x_{n k}$ & 1 & 2 & $\ldots$ & $\cdots$ & {$\left[\sqrt{\Delta_{01} h_{r s}}\right]$} & $\cdots$ \\
\hline 1 & 1 & 2 & $\cdots$ & $\cdots$ & $\sqrt{\Delta_{01} h_{r s}}$ & $\cdots$ \\
\hline 2 & 2 & $\ldots$ & $\ldots$ & $\cdots$ & $\ldots$ & $\cdots$ \\
\hline$\vdots$ & $\vdots$ & $\vdots$ & $\vdots$ & $\vdots$ & $\vdots$ & $\ldots$ \\
\hline$\vdots$ & $\vdots$ & $\vdots$ & $\vdots$ & $\vdots$ & $\vdots$ & $\ldots$ \\
\hline$\left[\sqrt{\Delta_{10} h_{r s}}\right]$ & {$\left[\sqrt{\Delta_{10} h_{r s}}\right]$} & $\cdots$ & $\cdots$ & $\cdots$ & $\cdots$ & $\cdots$ \\
\hline$\vdots$ & $\vdots$ & $\vdots$ & $\vdots$ & $\ldots$ & $\vdots$ & $\ldots$ \\
\hline$\vdots$ & $\vdots$ & $\vdots$ & $\vdots$ & $\ldots$ & $\vdots$ & $\ldots$ \\
\hline
\end{tabular}

Then for any $\varepsilon>0$,

$$
\begin{aligned}
& P-\frac{1}{\Delta h_{r s}} C\left(\left\{(n, k) \in \Delta I_{r s}:\left\|\Delta_{11} x_{n k}-\bar{\theta}\right\|_{E} \geq \varepsilon\right\}\right) \\
< & P-\frac{\left[\sqrt{\Delta_{01} h_{r s}}\right]\left[\sqrt{\Delta_{10} h_{r s}}\right]}{\Delta_{01} h_{r s} \Delta_{10} h_{r s}} \\
\longrightarrow & 0 \text { as } r, s \rightarrow \infty
\end{aligned}
$$

i.e., $x_{n k} \stackrel{P}{\longrightarrow} \bar{\theta}\left(\Delta_{11}-S_{\theta_{r, s}}\right)$.

But,

$$
\begin{aligned}
& P-\frac{1}{\Delta h_{r s}} \sum_{(n, k) \in \Delta I_{r s}}\left\|\Delta_{11} x_{n k}-\bar{\theta}\right\|_{E} \\
< & P-\frac{1}{\Delta h_{r s}} \frac{\left[\sqrt{\Delta_{01} h_{r s}}\right]\left(\left[\sqrt{\Delta_{01} h_{r s}}\right]+1\right)}{2} \times \frac{\left[\sqrt{\Delta_{10} h_{r s}}\right]\left(\left[\sqrt{\Delta_{10} h_{r s}}\right]+1\right)}{2} \\
\longrightarrow & \frac{1}{4} \neq 0 \text { as } r, s \rightarrow \infty
\end{aligned}
$$

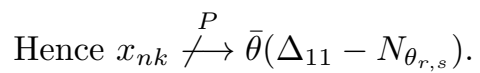

This shows that the converse is not true in general.

(b) Suppose $x_{n k} \stackrel{P}{\longrightarrow} \ell\left(\Delta_{11}-S_{\theta_{r, s}}\right)$ and $x=\left(x_{n k}\right) \in \ell_{\infty}\left(\Delta_{11}\right)$, say,

$$
\left\|\Delta_{11} x_{n k}-\ell\right\|_{E} \leq M \text { for all } \mathrm{n}, \mathrm{k} .
$$


Then for any given $\varepsilon>0$ and large $\mathrm{r} \& \mathrm{~s}$ we obtain the following

$$
\begin{array}{rl} 
& \frac{1}{\Delta h_{r s}} \sum_{\substack{(n, k) \in \Delta I_{r s} \\
<}}\left\|\Delta_{11} x_{n k}-\ell\right\|_{E} \\
+ & \frac{1}{\Delta h_{r s}} \sum_{\substack{(n, k) \in \Delta I_{r s} \\
\left\|\Delta_{11} x_{n k}-\ell\right\|_{E} \geq \varepsilon}}\left\|\Delta_{11} x_{n k}-\ell\right\|_{E} \\
\leq & \frac{1}{\Delta h_{r s}} \sum_{\substack{(n, k) \in \Delta I_{r s} \\
\left\|\Delta_{11} x_{n k}-\ell\right\|_{E}<\varepsilon}}\left\|\Delta_{11} x_{n k}-\ell\right\|_{E} \\
\leq h_{r s} & C\left(\left\{(n, k) \in \Delta I_{r s}:\left\|\Delta_{11} x_{n k}-\ell\right\|_{E} \geq \varepsilon\right\}\right)+\varepsilon
\end{array}
$$

Therefore $x_{n k} \stackrel{P}{\longrightarrow} \ell\left(\Delta_{11}-S_{\theta_{r, s}}\right)$.

Q.E.D

\section{Lacunary refinement}

Definition 5.1. Let $\theta_{r, s}=\left(k_{r s}\right)$ be a lacunary sequence. A lacunary double sequence $\theta_{r, s}^{\prime}=\left(k_{r s}^{\prime}\right)$ is said to be lacunary refinement of $\theta=\left(k_{r s}\right)$ if $\left(k_{r s}\right) \subseteq\left(\dot{k}_{r s}\right)$.

In the following theorem we will discuss the inclusion properties of different lacunary methods for double sequence and find out the general description of the inclusion between two arbitrary lacunary methods for double sequences.

Theorem 5.1. If $\theta_{r, s}^{\prime}$ is a lacunary refinement of $\theta_{r, s}$ and $x_{n k} \stackrel{P}{\longrightarrow} \ell\left(\Delta_{11}-S_{\theta_{r, s}^{\prime}}\right)$, then $x_{n k} \stackrel{P}{\longrightarrow}$ $\ell\left(\Delta_{11}-S_{\theta_{r, s}}\right)$.

Proof. Suppose each $\Delta I_{r s}$ of $\theta_{r, s}$ contains the points $\left\{k_{r(i), s(j)}^{\prime}\right\}_{i=1, j=1}^{\nu(r) \mu(s)}$ such that each $\Delta_{10} I_{r s}$ of $\theta_{r, s(\text { fixed })}$ contains the points $\left(k_{r(i), s(\text { fixed })}^{\prime}\right)_{i=1}^{\nu(r)}$ of $\theta_{r, s}^{\prime}$ so that

$$
k_{r-1, s}<k_{r(1), s}^{\prime}<k_{r(2), s}^{\prime}<\ldots<k_{r(\nu(r)), s}^{\prime}=k_{r, s}
$$

where

$$
\Delta I_{r(i), s(\text { fixed })}^{\prime}=\left(k_{r(i-1), s}^{\prime}, k_{r(i), s}^{\prime}\right], i=2, \ldots, \nu(r)
$$

while each $\Delta_{01} I_{r s}$ of $\theta_{r(\text { fixed }), \mathrm{s}}$ contains the points $\left(k_{r(\text { fixed }), \mathrm{s}(\mathrm{j})}^{\prime}\right)_{j=1}^{\mu(s)}$ of $\theta_{r, s}^{\prime}$ so that

$$
k_{r, s-1}<k_{r, s(1)}^{\prime}<k_{r, s(2)}^{\prime}<\ldots<k_{r, s(\mu(s)}^{\prime}=k_{r, s}
$$

where

$$
\Delta I_{r(\text { fixed }), \mathrm{s}(\mathrm{j})}^{\prime}=\left(k_{r, s(j-1)}^{\prime}, k_{r, s(j)}^{\prime}\right], j=2, \ldots, \mu(s) .
$$

Clearly for all $\mathrm{r}$ and $\mathrm{s}, \nu(r) \geq 1, \mu(s) \geq 1$, because $\left\{k_{r s}\right\} \subseteq\left\{k_{r s}^{\prime}\right\}$. So,

$$
\Delta I_{r(i), s(j)}^{\prime}=\Delta I_{r(i), s(\text { fixed })}^{\prime} \times I_{r(\text { fixed }), \mathrm{s}(\mathrm{j})}^{\prime} .
$$


Let $\left\{\Delta I_{p, q}^{*}\right\}_{p, q=1,2, \ldots}$ be the sequence of abutting intervals $\left\{\Delta I_{r(i), s(j)}^{\prime}\right\}$ ordered by increasing right end points $k_{r(i), s \text { (fixed) }}^{\prime}$ of $\Delta_{10} I_{r(i), s}^{\prime}$ and right end points $k_{r(\text { fixed }), \mathrm{s}(\mathrm{j})}^{\prime}$ of $\Delta_{01} I_{r, s(j)}^{\prime}$.

Since $x_{n k} \stackrel{P}{\longrightarrow} \ell\left(\Delta_{11}-S_{\theta_{r, s}^{\prime}}\right)$ we get for each $\varepsilon>0$,

$$
P-\lim _{p, q} \sum_{\Delta I_{p, q}^{*} \subseteq \Delta I_{r, s}} \frac{1}{\Delta h_{r s}^{*}} C\left(\left\{(n, k) \in \Delta I_{p, q}^{*}:\left\|\Delta_{11} x_{n k}-\ell\right\|_{E} \geq \varepsilon\right\}\right)=0 .
$$

As before we write,

$$
\begin{aligned}
\Delta h_{r s} & =\Delta_{10} h_{r, s} \times \Delta_{01} h_{r, s} \\
& =\left(k_{r, s}-k_{r-1, s}\right) \times\left(k_{r, s}-k_{r, s-1}\right) \\
\Delta h_{r s}^{\prime}= & \Delta_{10} h_{r(i), s}^{\prime} \times \Delta_{01} h_{r, s(j)}^{\prime} \\
= & \left(k_{r(i), s}^{\prime}-k_{r(i-1), s}\right) \times\left(k_{r, s(j)}-k_{r, s(j-1)}\right)
\end{aligned}
$$

and

$$
\Delta_{10} h_{r(i), s}^{\prime}=k_{r(i), s}^{\prime}-k_{r(i-1), s}
$$

and

$$
\Delta_{01} h_{r, s(j)}^{\prime}=k_{r, s(j)}-k_{r, s(j-1)}
$$

For each $\varepsilon>0$, we have

$$
\begin{aligned}
& \frac{1}{\Delta h_{r s}} C\left(\left\{(n, k) \in \Delta I_{r s}:\left\|\Delta_{11} x_{n k}-\ell\right\|_{E} \geq \varepsilon\right\}\right) \\
= & \frac{1}{\Delta h_{r s}} \sum_{I_{p q}^{\star} \subseteq \Delta I_{r s}} \Delta h_{p q}^{\star} \frac{1}{\Delta h_{p q}^{\star}} C\left(\left\{(n, k) \in \Delta I_{p q}^{\star}:\left\|\Delta_{11} x_{n k}-\ell\right\|_{E} \geq \varepsilon\right\}\right) \\
= & \frac{1}{\Delta h_{r s}} \sum_{\Delta I_{p q}^{\star} \subseteq \Delta I_{r s}} \Delta h_{p q}^{\star}\left(C_{\dot{\theta}} \chi_{\mathbf{K} \times \mathbf{K}}\right)_{p, q}
\end{aligned}
$$

where $\chi_{\mathbf{K} \times \mathbf{K}}$ is the characteristic function of the set

$$
K \times K=\left\{(n, k) \in N \times N:\left\|\Delta_{11} x_{n k}-\ell\right\|_{E} \geq \varepsilon\right\}
$$

and $C_{\hat{\theta}} \chi_{\mathbf{K} \times \mathbf{K}}$ is defined by

$$
\left(C_{\dot{\theta}} \chi_{\mathbf{K} \times \mathbf{K}}\right)_{p, q}=\frac{C\left((K \times K) \cap \Delta I_{p q}^{\star}\right)}{\Delta h_{p q}^{\star}}
$$

By (5.2), $C_{\dot{\theta}} \chi_{\mathbf{K} \times \mathbf{K}}$ is a null sequence and (5.3) is a regular weighted mean transform of $C_{\hat{\theta}} \chi_{\mathbf{K} \times \mathbf{K}}$. Hence the transform (5.3) also tends to zero as r, s $\rightarrow \infty$. i.e., $x_{n k} \stackrel{P}{\longrightarrow} \ell\left(\Delta_{11}-S_{\theta_{r, s}}\right)$ Q.E.D.

Next theorem gives the converse. 
Theorem 5.2. Let $\theta_{r, s}^{\prime}=\left(\dot{k}_{r s}\right)$ be a lacunary refinement of lacunary sequence $\theta_{r, s}=\left(k_{r s}\right)$. Let $\Delta I_{r s}$ and $\Delta I_{r s}^{\prime}, r, s=1,2, \ldots$ be the corresponding intervals of $\theta_{r, s}$ and $\theta_{r, s}^{\prime}$ respectively and $\Delta h_{r s}$ and $\Delta h_{r s}^{\prime}$ are $\Delta_{10} h_{r, s} \times \Delta_{01} h_{r, s}$ and $\Delta_{10} h_{r, s}^{\prime} \times \Delta_{01} h_{r, s}^{\prime}$ respectively. If there exist $\delta>0$ such that

$$
\frac{\Delta \hat{h}_{r s}}{\Delta h_{i j}} \geq \delta, \text { for every } \Delta I_{r s}^{\prime} \subseteq \Delta I_{i j},
$$

then $x_{n k} \stackrel{P}{\longrightarrow} \ell\left(\Delta_{11}-S_{\theta_{r, s}}\right)$ implies $x_{n k} \stackrel{P}{\longrightarrow} \ell\left(\Delta_{11}-S_{\theta_{r, s}^{\prime}}\right)$.

Proof. Let $\varepsilon>0$ be given.

Then for every $\Delta I_{r s}^{\prime}$, we can find $\Delta I_{i j}$ such that $\Delta I_{r s}^{\prime} \subseteq \Delta I_{i j}$.

Then we have

$$
\begin{aligned}
& \frac{1}{\Delta \hat{h}_{r s}} C\left(\left\{(n, k) \in \Delta I_{r s}^{\prime}:\left\|\Delta_{11} x_{n k}-\ell\right\|_{E} \geq \varepsilon\right\}\right) \\
= & \left(\frac{\Delta h_{i j}}{\Delta \hat{h}_{r s}}\right)\left(\frac{1}{\Delta h_{i j}}\right) C\left(\left\{(n, k) \in \Delta I_{r s}^{\prime}:\left\|\Delta_{11} x_{n k}-\ell\right\|_{E} \geq \varepsilon\right\}\right) \\
\leq & \left(\frac{\Delta h_{i j}}{\Delta \hat{h}_{r s}}\right)\left(\frac{1}{\Delta h_{i j}}\right) C\left(\left\{(n, k) \in \Delta I_{i j}:\left\|\Delta_{11} x_{n k}-\ell\right\|_{E} \geq \varepsilon\right\}\right)\left(\text { since } \Delta I_{r s}^{\prime} \subseteq \Delta I_{i j}\right) \\
\leq & \left(\frac{1}{\delta}\right)\left(\frac{1}{\Delta h_{i j}}\right) C\left(\left\{(n, k) \in \Delta I_{i j}:\left\|\Delta_{11} x_{n k}-\ell\right\|_{E} \geq \varepsilon\right\}\right) .
\end{aligned}
$$

Q.E.D.

Remark. This theorem establishes inclusion between two lacunary methods only when one sequence is a refinement of the other.

Next theorem gives the converse in general.

Theorem 5.3. Suppose $\theta_{r, s}^{\prime}=\left(\dot{k}_{r s}\right)$ be a lacunary refinement of lacunary sequence $\theta_{r, s}=\left(k_{r s}\right)$. Let $\Delta I_{r s}$ and $\Delta I_{r s}^{\prime}, r, s=1,2, \ldots$ be the corresponding intervals of $\theta$ and $\theta$ respectively and $\Delta P_{r s i j}=$ $\Delta I_{r s}^{\prime} \cap \Delta I_{i j}, r, s, i, j=1,2, \ldots$. If there exist a $\delta>0$ such that

$$
\frac{\Delta h_{r s i j}}{\Delta h_{i j}} \geq \delta, \text { for every } r, s, i, j=1,2, \ldots, \text { provided } \Delta P_{r s i j} \neq \varphi
$$

Then $x_{n k} \stackrel{P}{\longrightarrow} \ell\left(\Delta_{11}-S_{\theta_{r, s}}\right)$ implies $x_{n k} \stackrel{P}{\longrightarrow} \ell\left(\Delta_{11}-S_{\theta_{r, s}^{\prime}}\right)$.

Proof. Let $\alpha_{r, s}=\theta_{r, s} \cup \theta_{r, s}^{\prime}$. Then $\alpha$ is a refinement of the lacunary sequence $\theta_{r, s}$ and $\theta_{r, s}^{\prime}$. The interval determined by $\alpha_{r, s}$ is denoted by $\Delta P_{r s i j}=\Delta I_{r s}^{\prime} \cap \Delta I_{i j}, \Delta P_{r s i j} \neq \varphi$.

Now,

$$
\begin{aligned}
& \frac{1}{\Delta h_{r s i j}} C\left(\left\{(n, k) \in \Delta P_{r s i j}:\left\|\Delta_{11} x_{n k}-\ell\right\|_{E} \geq \varepsilon\right\}\right) \\
= & \left(\frac{\Delta h_{i j}}{\Delta h_{r s i j}}\right)\left(\frac{1}{\Delta h_{i j}}\right) C\left(\left\{(n, k) \in \Delta P_{r s i j}:\left\|\Delta_{11} x_{n k}-\ell\right\|_{E} \geq \varepsilon\right\}\right) \\
\leq & \left(\frac{\Delta h_{i j}}{\Delta h_{r s i j}}\right)\left(\frac{1}{\Delta h_{i j}}\right) C\left(\left\{(n, k) \in \Delta I_{i j}:\left\|\Delta_{11} x_{n k}-\ell\right\|_{E} \geq \varepsilon\right\}\right) \\
\leq & \left(\frac{1}{\delta}\right)\left(\frac{1}{\Delta h_{i j}}\right) C\left(\left\{(n, k) \in \Delta I_{i j}:\left\|\Delta_{11} x_{n k}-\ell\right\|_{E} \geq \varepsilon\right\}\right)
\end{aligned}
$$


Hence $x_{n k} \stackrel{P}{\longrightarrow} \ell\left(\Delta_{11}-S_{\theta_{r, s}}\right)$ implies $x_{n k} \stackrel{P}{\longrightarrow} \ell\left(\Delta_{11}-S_{\alpha_{r, s}}\right)$.

By Theorem 5.1 it follows that $x_{n k} \stackrel{P}{\longrightarrow} \ell\left(\Delta_{11}-S_{\alpha_{r, s}}\right)$ implies $x_{n k} \stackrel{P}{\longrightarrow} \ell\left(\Delta_{11}-S_{\theta_{r, s}^{\prime}}\right)$, since $\alpha_{r, s}$ is a lacunary refinement of $\theta_{r, s}^{\prime}$.

Q.E.D.

Similarly if we change the given condition into $\frac{\Delta h_{r s i j}}{\Delta h_{r s}} \geq \delta$, for every $r, s, i, j=1,2, \ldots$, provided $\Delta P_{r s i j} \neq \varphi$, then we get $x_{n k} \stackrel{P}{\longrightarrow} \ell\left(\Delta_{11}-S_{\theta_{r, s}^{\prime}}\right)$ implies $x_{n k} \stackrel{P}{\longrightarrow} \ell\left(\Delta_{11}-S_{\theta_{r, s}}\right)$.

Combining this condition and Theorem 5.3, we get the following Theorem 5.4, which gives the sufficient conditions to have the relationship between two arbitrary lacunary methods.

Theorem 5.4. Suppose $\theta_{r, s}=\left(k_{r s}\right)$ and $\theta_{r, s}^{\prime}=\left(k_{r s}\right)$ be two lacunary double sequences. Let $\Delta I_{r s}$ and $\Delta I_{r s}^{\prime}$ be the corresponding intervals of $\theta_{r, s}$ and $\theta_{r, s}^{\prime}$ respectively and $\Delta P_{r s i j}=\Delta I_{r s}^{\prime} \cap \Delta I_{i j}$, $r, s, i, j=1,2, \ldots$. If there exist a $\delta>0$ such that

$$
\frac{\Delta h_{r s i j}}{\left(\Delta h_{i j}+\Delta h_{r s}\right)} \geq \delta, \text { for every } i, j, r, s=1,2, \ldots, \text { provided } \Delta P_{r s i j} \neq \varphi
$$

Then $x_{n k} \stackrel{P}{\longrightarrow} \ell\left(\Delta_{11}-S_{\theta_{r, s}}\right)$ implies and is implied by $x_{n k} \stackrel{P}{\longrightarrow} \ell\left(\Delta_{11}-S_{\theta_{r, s}^{\prime}}\right)$.

\section{Summary and Conclusion}

In this paper, we have extended the notion of lacunary convergence from single sequence of scalars to double sequence of scalars. Further, the space $N_{\theta}$ of Freedman et al. [4] has been extended to $\Delta_{11} N_{\theta_{r, s}}(E)$, the space of lacunary strongly convergent vector valued double sequences. Moreover, $\Delta_{11}$-statistical convergence, $\Delta_{11}$-lacunary statistical convergence and the concept of $\Delta_{11}$-statistical Cauchy sequence are introduced in these new classes of vector valued double sequence spaces. Some inclusion relations for lacunary refinements are derived. The corresponding results of Jinlu Li [20] can be derived from our results.

\section{References}

[1] A. Basu and P. D. Srivastava, $\Delta$-lacunary strong A-convergent vector valued difference sequences with respect to a sequence of Orlicz functions and some inclusion relations, Int. J. Pure Appl. Math., 11 (3) (2004), 335-353.

[2] A. Basu and P. D. Srivastava, Generalized vector valued double sequence space using modulus function, Tamkang Journal of Mathematics, 38(4) (2007), 347-366.

[3] A. Pringsheim, Zur Theorie der zweifach unendlichen Zahlenfolgen, Math. Ann. 53 (1900), 289-321.

[4] R. Allen, John J. Freedman and M.R. Sember, Some Cesaro type Summability spaces, Proc. London math. Soc. 37(3) (1978), 508-520.

[5] A. Zygmund, Trigonometric series, Cambridge, UK, Cambridge University Press, (1979)

[6] D. Rath and B. C. Tripathy, On statistically convergent and statistically Cauchy sequences, Indian Jour. Pure Appl. Math., 25(4), (1994), 381-386.

[7] E. Savas and R. F. Patterson, Some double lacunary sequence spaces by Orlicz funcion, Southeast Asian Bull Math, 35 (1), (2011), 103-110.

[8] H. Çakalli, Lacunary statistical convergence in topological groups, Indian J. Pure Appl. Math., 26(2) (1995), 113-119. 
[9] J. S. Connor, The statistical and strong p-Cesaro convergence of sequences, Analysis, 8 (1988), 47-63.

[10] J. A. Fridy and C. Orhan, Lacunary statistical convergence, Pacific J. Math., 160(1) (1993), 43-51.

[11] J. A. Fridy and C. Orhan, Lacunary statistical summability, J. Math. Anal. Appl., 173 (1993), 497-504.

[12] V. Karkaya and N. Şimşek, On Lacunary invariant sequence spaces defined by a sequence of modulus function, Appl. Math. Comp., 156 (3), (2004), 597-603.

[13] E. Mursaleen and H. H. Osama, Statistical convergence of double sequences, J. Math. Anal. Appl., 288 (2003) 223-231.

[14] H. Fast, Sur la convergence statistique, Collog. Math., 2 (1951), 241-244.

[15] I. J. Schoenberg, The integrability of certain functions and related summability methods, Amer. Math. Monthly, 66 (1959), 261-375.

[16] I. Niven and H. S. Zuckerman, An introduction to the theory of numbers, Fourth Edi., John Wiley \& Sons, New York, 1980.

[17] I. J. Maddox, Spaces of strongly summable sequences, Quart. J. Math. Oxford, 18 (2) (1967), 345-355.

[18] J. Connor, The statistical and strong p-Cesaro convergence of sequence, Analysis, 8 (1988), 47-63.

[19] J. A. Fridy, On statistical convergence, Analysis, 5 (1985), 301-313.

[20] Jinlu Li, Lacunary statistical convergence and inclusion properties between lacunary methods, Inter. J. Math. and Math. Sci., 23(3) (2000), 175-180.

[21] O. H. H. Edely and M. Mohammad, Tuberian theorems for statistically convergent double sequences, Information Sciences, 176 (2006), 875-886.

[22] R. C. Buck, Generalized asymptotic density, Amer. J. Math., 75 (1953), 335-346.

[23] T. S̆alát, On statistically convergent sequences of real numbers, Math. Slovaca, 30 (1980), 139-150.

[24] T. Bilgin, Lacunary strong A-convergence with respect to a sequence of Modulus functions, Appl. Math. Comput., 151(3) (2004), 595-600. 